\title{
Classification of Name Based On Leaf Recognition Using BT and ED Algorithm
}

\author{
A.M.Ravishankkar \\ Karpagam University, \\ Coimbatore,Tamilnadu
}

India

\author{
M.Mohanapriya, \\ Karpagam University, \\ Coimbatore, Tamilnadu, \\ India
}

\begin{abstract}
:
The main purpose of this paper should be to show that the outer frame of a leaf and with the help of Back propagation Network is enough to give a reasonable statement about the species category is identified. Leaves Recognition is a neuronal network based java application/applet to recognize images of leaves using Back propagation Network. The intention is to give the user the ability to administrate a hierarchical list of images, where they can perform some sort of image using edge detection to identify the individual tokens of every image. The Thinning algorithm here is used to process the image recursively and minimizes the found lines to a one-pixel wide one by comparing the actual pixel situation with specific patterns can be identified and then minimizes it. The urgent situation is that due to environmental degradation and lack of awareness, many rare plant species are at the risk of extinction so it is necessary to keep record for plant protection. It focuses on using digital image processing for the purpose of automate classification and recognition of plants based on the images of the leaves. It help to protect the plant and mainly it is used for highly production of rare plant or herbal plant used for medical purpose. Efficacy of the proposed methods is studied by using two neural classifiers. These are neuro-fuzzy controller and a feed-forward back-propagation multi-layered perception to discriminate between 28 classes of leaves. The features have been applied individually as well as in combination to investigate how recognition accuracies can be improved with the help of B\&T algorithm.
\end{abstract}

Keywords: Image Processing, Edge detection, Neuronal network ,thinning algorithm, B\&T algorithm.

\section{INTRODUCTION:}

Plants play the most important part in the life cycle of nature. They are the primary producers that sustain all other life forms including animal, people and also Non living things. This is because only plants are the only organisms or species that can convert light energy from the sun into food. Both human and animals are incapable of making their own food, depend directly or indirectly on plants for their supply of food. Leaves of same species also have variation in there shapes and moreover leaves of different species may have a same size because of the complex nature of leaves. So it is very difficult to identify plant name for that we need higher process of computing the leaves with efficient technique. A leaf from an unknown species of plant will be the input to the proposed system and trained set is to identify plant recognition scheme based on the trained system.To handle such volumes of information ,development of a quick and efficient classification and decision based method has become an area of active research. In nature, plant leaves are two dimensional containing important features that can be useful for classification of various plant species, such as shapes, colours, textures and structures of their leaf can be varied from one to another [1,4]. A leaf from an unknown species of plant will be the input to the proposed system. The system then segments the leaf image from its background, computes the 
morphological feature representation help for matching the leaf, and then displays the similarity percentage as computed [5]. The leaf image will be captured on a plain contrast background to reduce the complexity of the segmentation algorithm and give better performance.

The present paper proposes a scheme for automated recognition of three types of plant species by analyzing shape features from digital images of their leaves with the help of enhanced algorithm. It

\section{RELATED WORK:}

Beside the fact of writing a java based application to realize this purpose, one additional feature is that it could also be used as a java applet to directly give the user the ability to start it via a java enabled internet browser. The main tasks of this application is used to detect the tokens using prewitt edge detection algorithm[2].A plant leaf identification, most of them used neuronal network algorithm. Image processing is most important preliminary phase and it taking image as a tokens.. This tokens will then be the basis of the neuronal network calculations to make it possible to recognize a unknown leaf image and specify the species it belongs to.This paper implements a leaf recognition algorithm using easy-to-extract features and high efficient recognition algorithm. Our main improvements are on feature extraction and the classifier. All features are extracted from digital leaf image. Except one feature, all features can be extracted automatically[7]. Jyotismita Chaki, Ranjan Parekh [9].In this paper leaf recognition has been done by using shape analysis and feature extraction. With the help of netural network which under supervised learning algorithm having multilayer preceptor weight can be verified with feed forward backward back propagation architecture.

Most popular algorithm to identify name for the plant with the help of leaf. General regression neural networks perform regression where the target Variable of the two values is continuous. The main aim is to predicted target value of an item(leaf) is similar to be the same as other items(leaf) that have close values of the predictor variables. The k-nearest neighbor algorithm is the most popular algorithms is to identify object (leaf) which is common to all of the other leaf have a similar properties to follows: Section 2 provides an overview of related work, Section 3 outlines the proposed approach with discussions on overview, feature computation and classification schemes, Section 4 provides details of the dataset and experimental results obtained and Section 5 provides the overall conclusion and the scope for future research.

match with another. Gabor wavelet/AN Networks system to classify images texture to identify the name of the plant . K-means clustering is an algorithm to classify the objects based on attributes/features into $K$ number of groups where $K$ is a positive integer. $\mathrm{K}$ - means clustering is a supervised learning algorithm and it have a prior knowledge of the number of clusters maximizing intra clustering and Minimizing inter clustering.

In the neuronal network is used for sigmoid function[12].Plant species identification requires recognizing the plant by various characteristics, such as size, form, leaf shape, flower color, odor, etc., and linking it with a common or so-called scientific name[8]. The classification algorithm implemented for accurate identification of the plants based on Leaf image. Different data modelling techniques used include curvature scale space, fuzzy logic, fractal dimensions[9], Fourier analysis ,wavelets [9,11], curvelets and Zernike moments . A variety of classifiers have also been used viz. neural networks [3], support vector machines , nearest neighbours [6], and K-means for identifying unknown leaves[10].

\section{PROPOSED SYSTEM:}

Our system is based on image processing which finds an unknown leaf species without any previous knowledge, which is useful for any layman. The basic factors for identification of species are, image edge detection, back propagation and Neural Network shown in Fig 1.It is used to retrieval of leaf images based on the shape of the leaf image given as input by the user. For example, If the input is a unknown leaf's image, then the output will be given that determine the which leaf is present here.

\subsection{Converting RGB image to binary image}


The leaf image is acquired by scanners or digital cameras. Since we have not found any digitizing device to save the image in a lossless compression format, the image format here is JPEG. All leaf images are in $800 \times 600$ resolution better for identify for the purpose. There is no restriction on the direction of leaves when photoing.

In this approach we follows several steps,

1. user uploads an image of leaf of his /her choice.

2.we find out the feature extraction using image preprocessing.

3. we find out the image edge detection and thinning algorithm.

4.comparing the training data set with test data set.

5.Then determine the information about the particular image.

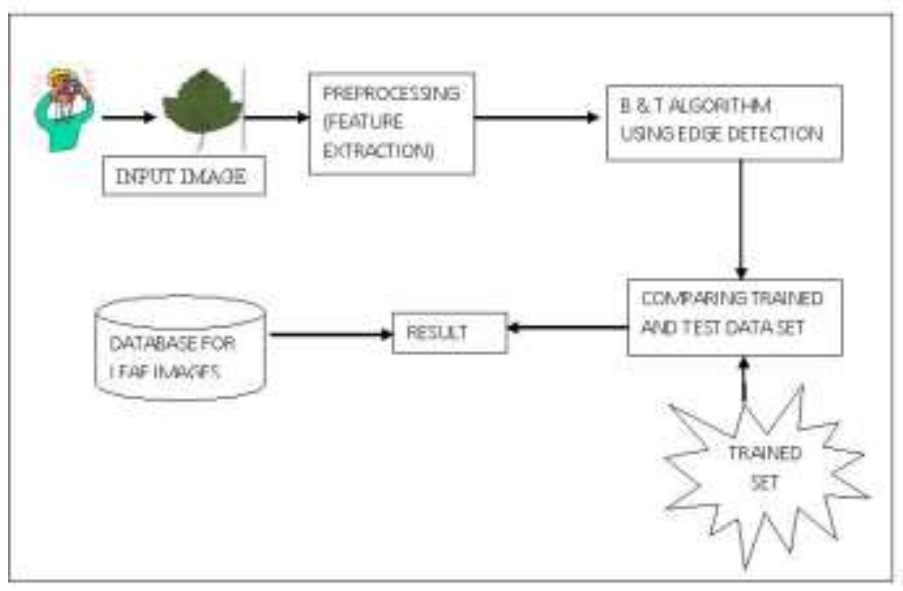

Fig 1.Architecture Diagram

STEP 1: Image Upload

User can upload any image of a leaf in jpeg or png format of his or her choice. We have ready dataset for comparing with that unknown image.

\section{STEP 2: Image Preprocessing}

Image processing is the enhancement of image i.e., processing an image so that the results are suitable for particular application. Processing image involves operations for making the background white for removing unwanted noise.One of the most important parts of the whole application is the image processing. Without finding any usefull tokens in the leaf images, the neuronal network calculation. So we spent lots of our efforts in the edge detection and thinning algorithms.

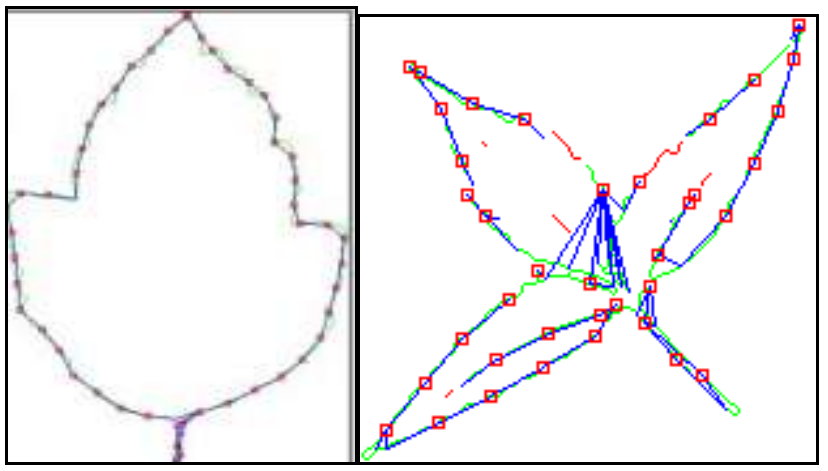

Fig 2 Image edge detection

In edge detection, the distance of the tokens (red square) and a minimum amount of pixels a line have to be to be recognized as a part of the shape. For a good quality result you should normally add at least 5 images of a species to give the neuronal network enough tokens to find the specific shape of this leaf species.

\section{STEP 3: Edge Detection}

The proposed system finds the specific tokens which represents that portion of the image where transfer of lower to higher pixel intensity occurs. These tokens are used for neural network calculations. Edges are recognized by abrupt intensity variations in an image. The first gradient is typically calculated to locate the edges. The $3 * 3$ masks are used to extract the edges from image and convolved them through it. Prewitt edge detection technique is selected due to efficiency and simplicity in single template.

Prewitt edge detection produces an image (Fig2) where higher grey-level values indicate the presence of an edge between two objects. The Prewitt Edge Detection filter computes the root mean square of two $3 \times 3$ templates. It is one of the most popular $3 \times 3$ edge detection filters. The Prewitt edge detection filter uses these two $3 \times 3$ templates to calculate the gradient value:

$$
\begin{array}{llllll}
-1 & 0 & 1 & 1 & 1 & 1
\end{array}
$$




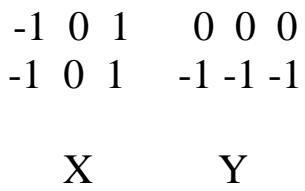

Now consider the following $3 \times 3$ image window:

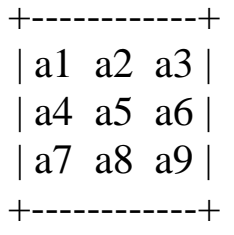

where:

- a1 .. a9 - are the grey levels of each pixel in the filter window

- $\mathrm{X}=-1 * \mathrm{a} 1+1 * \mathrm{a} 3-1 * \mathrm{a} 4+1 * \mathrm{a} 6-$ $1 * \mathrm{a} 7+1 * \mathrm{a} 9$

- $\mathrm{Y}=1 * \mathrm{a} 1+1 * \mathrm{a} 2+1 * \mathrm{a} 3-1 * \mathrm{a} 7-1 * \mathrm{a} 8$ $-1 * a 9$

- Prewitt gradient $=\mathrm{SQRT}\left(\mathrm{X}^{*} \mathrm{X}+\right.$ $\left.\mathrm{Y}^{*} \mathrm{Y}\right)$

All pixels are filtered. In order to filter pixels located near the edge of an image, edge pixels values are replicated to give sufficient data. The idea behind the transfer of the leaf image shape into a neuronal network usable form is, that the cosines and sinus angles of the shape represents the criteria of a recognition pattern.

The right hand image shows a part of a leaf image that was already processed through the above mentioned edge detection and thinning algorithms.

To give you an idea of what you see in this image,

- Green line: The shape of the leaf image after successful edge detection \& thinning.

- Red Square: This square represents a point on the shape of the leaf image from which we are going to draw a line to the next square.

- Blue line: The compound of the center of two squares from which we are going to calculate the cosines and sinus angle. Such a blue line is a representation of a leaf token.
Mainly this configuration is the properties of the neuronal network. It based on the amount of images and network properties you normally need to specify around 500-1000 training steps to get a good result in the recognition later. If the error rate drops below 0.01 you normally should encounter no problem in recognizing different leaf images.

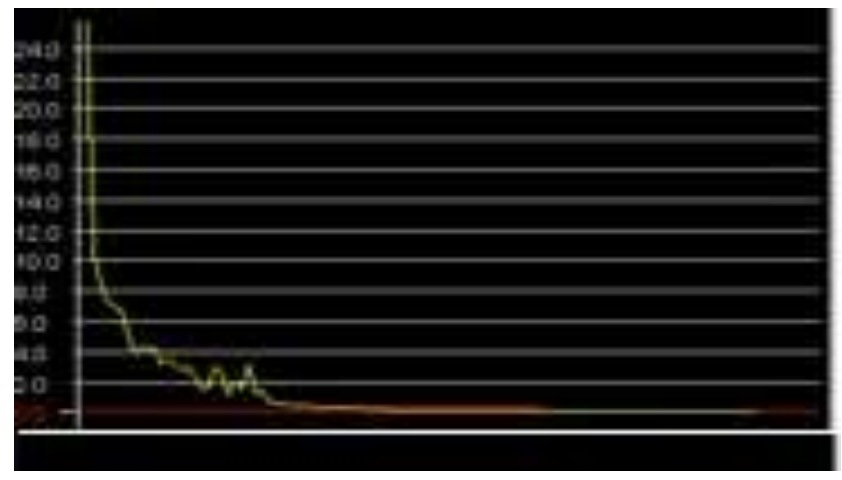

Fig 3 Detection graph

\section{STEP 4: Neuronal network}

Another main part of this work is the integration of a feed-forward back propagation neuronal network. As described earlier the inputs for this neuronal network are the individual tokens of a leaf image, and as a token normally consists of a cosinus and sinus angle, the amount of input layers for this network are the amount of tokens multiplied by two. The number of output neurons is normally specified by the amount of different species because we use a encoded form to specify the outputs. All other behaviour of the network is specified by the normal mathematical principals of a back propagation network. This neuronal network adopts with three tier network structure including the input layer, hidden layer[7], output layer.

In our system we are using neural network with one hidden layer. Each hidden layer is associated with the sigmoid function. In other words neurons in a same layer have same activation function. Sigmoid function is an exponential function which is used for calculation and transfer of knowledge from input neurons to output neurons. The graph for sigmoid function can be shown as in Fig 3. 


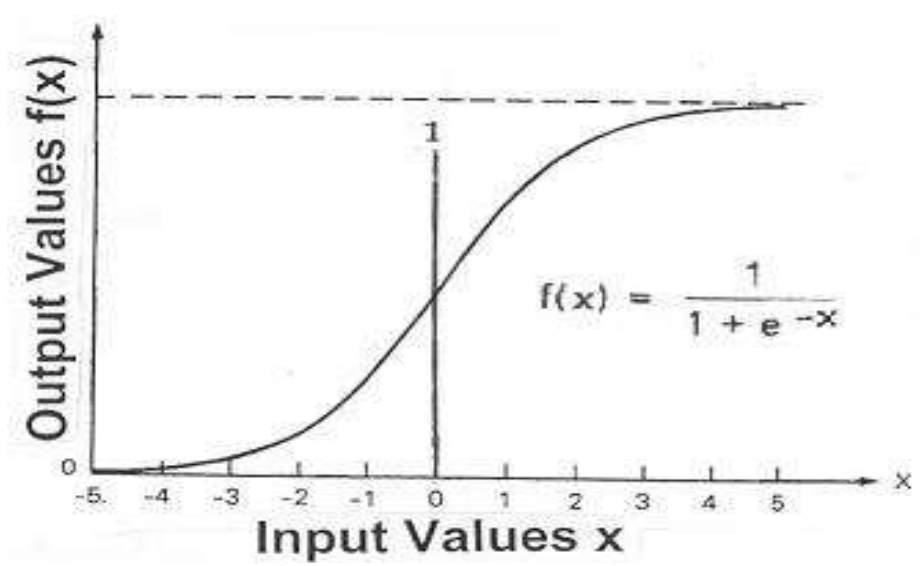

Fig 3 Sigmoid function

Sigmoid Function: $\mathrm{f}(\mathrm{x})=1 /\left(1+\mathrm{e}^{\wedge}-\mathrm{x}\right)$

Sigmoid Derivative: $\mathrm{f}^{\prime}(\mathrm{x})=\mathrm{f}(\mathrm{x}) *[1-\mathrm{f}(\mathrm{x})]$

\section{STEP 5: Thinning}

To identifying a specific leaf image's species here is that the outer frame of a leaf is enough to specify the species it belongs to. To accomplish that, it is necessary to identify this outer frame exactly. The previously applied Prewitt Edge detection normally just identifies the edges with a preconfigured threshold and after this edge detection we have to perform a thinning algorithm to minimize this threshold-based edge to a one-line frame.

The used thinning algorithm here processed the image recursively and minimizes the found lines to a one-pixel wide one by comparing the actual pixel situation with specific patterns and then minimizes it.

\section{EXPERIMENTAL RESULT:}

We have used the dataset for various leaf species like Azadirachta indica(neem), Pinus(pine tree), Quercus(oak), etc . Here, Firstly we add new species then add images of the same species under it and find the tokens for each leaf image after that we proceed by training these tokens using neural network later in recognition panel we add the unknown leaf to be recognized.

In order to optimize obtained results, we used to combine these features, where we get more efficiency in classification; the following table and figure prove this idea.

\begin{tabular}{|c|c|c|c|c|}
\hline Algontums & Accuray & & & asue \\
\hline naive bays & 85.21 & 85.45 & 83.87 & 81.67 \\
\hline deosisontree & 68.88 & 56,09 & 87.45 & 45,78 \\
\hline menittedge detetion & 88.34 & 78.9 & 79.23 & 82,32 \\
\hline beckpropagation/thinning & 90,45 & 92.78 & 91.1 & 92,45 \\
\hline
\end{tabular}

Table 1 Results obtained by classification of edge detection algorithm

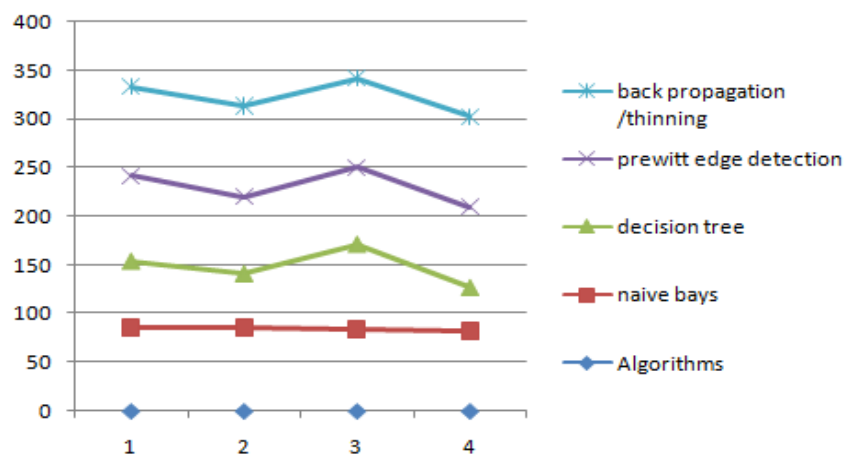

Fig 4 Results obtained by classification of edge detection algorithm

We compared the accuracy of our algorithm with other general purpose (not only applicable to certain species) classification algorithms that only use leafshape information. According to Table 1, the accuracy of our algorithm is very similar to other schemes. Considering our advantage respect to other automated/semi-automated general purpose schemes, easy-to- implement framework and fast speed of B \& $\mathrm{T}$ algorithm, the performance is very good.

Species Details

Botaical Narie : Thescer acorelolia

Mrathi tone i gatwo

\section{FATISP}

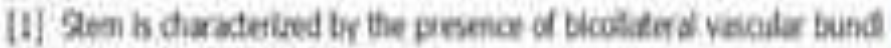
is sumounded by pericode fibres

[2] The whole biant indudeg root, stent and keves are uned to make medine

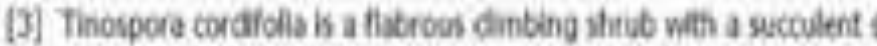
ten and papary hak the is cewwr white to grey in colout 


\section{CONCLUSION:}

Plants play an important role in our lives, without plants there will not be the existence of the ecology of the earth. The large amount of leaf types now makes the human being in a front of some problems in the specification of the use of plants, the first need to know the use of a plant is the identification of the plant leaf.The above analysis and graph, we display various details of unknown species in a specified area. For future scope diseases occurring in the unknown species and solution to overcome the diseases will provided and high production of rare plant. Back Propagation and Thinning algorithm must be done which gives output in terms of very high accuracy using minimal computational resources.

\section{REFERENCES}

[1] Jyotismita Chaki, Ranjan Parekh.:'Plant Leaf Recognition using Shape based Features and Network classifiers',(IJACSA) International Journal of Advanced Computer Science and Applications, Vol. 2, No. 10, 2011.

[2] Stephen Gang Wu, Forrest Sheng Bao, Eric You Xu, Yu- Xuan Wang, Yi-Fan Chang and QiaoLiang Xiang.:'A Leaf Recognition Algorithm for Plant Classification Using Probabilistic Neural Network', arXiv:0707.4289v1 [cs.AI] 29 Jul 2007.

[3] J. Chaki, and R. Parekh.:'Plant leaf recognit ion using shape based features and neural network classifiers', Internat ional Journal of Advanced Computer Science and Applications (IJACSA), 2011, pp.26-29.

[4] Sandeep Kumar's.:'Leaf color, area and edge Features based approach for Identification of Indian Medicinal plants',IJCSE Vol 3 No.3, 2012.

[5] S. Beucher, F. Meyer.:'The morphological approach to segmentation: the watershed transformation', E.R. Dougherty (Ed.), Mathematical Morphology in Image Processing, Marcel Dekker, 1993

[6] X.F. Wang, J.X. Du, G.J. Zhang.:' Recognition of leaf images based on shape features using a hypersphere classifier', Computer Sci. 3644(2005) 87-96.

[7] Mokhtarian, F., \& Abbasi, S.:'Matching shapes with self-intersections:Application to leaf classification', IEEE Transactions on Image Processing, 13(5), 653-661,2004.

[8] Lee, C.-L., \& Chen, S.-Y.:'Classification of leaf images', International Journal of Imaging Systems and Technology, 16(1), 15-23 (2006).

[9] X. Gu et al.,Wang.:'Leaf recognition based on the combination of wavelet transform and Gaussian interpolation', ICIS, vol. 3644/2005, pp. 253-262, 2005.

[10] Biva shrestha" classification of plants using images of their leaves" IJCSITS, Vol. 2 No. 2, 2012

[11] M. T. Hagan, H. B. Demut, and M. H. Beale, Neural Network Design, 2002

[12] Casanova, D., de Mesquita Sá Junior, J. J., Bruno, O. M.:'Plant leaf identification using gabor wavelets', International Journal of Imaging Systems and Technology, 19(3), 236-243.

[13] Sigmoid Function:

http://www.molecularcancer.com

[14] H. QI and J.-G. YANG.:'Sawtooth feature extraction of leaf edge based on support vector machine', Second International Conference on Machine Learning and Cybernetics, November 2003.

[15] S. M. Hong, B. Simpson, and G. V. G. Baranoski.:'Interactive venation- based leaf shape modeling', Computer Animation and Virtual Worlds, vol. 16, 2005.

[16] F. Gouveia, V. Filipe, M. Reis, C. Couto, and J. Bulas-Cruz.:'Biometry: the characterisation of chestnut-tree leaves using computer vision', IEEE International Symposium on Industrial Electronics, Guimarães, Portugal, 1997.

[17] X. Gu, J.-X. Du, and X.-F. Wang.:'Leaf recognition based on the combi- nation of wavelet transform and gaussian interpolation', International 
Conference on Intelligent Computing 2005, ser.

LNCS 3644. Springer, 2005.

[18] D. F. Specht.:'Probabilistic neural networks', Neural Networks, vol. 3, 1990.
[19] R. C. Gonzalez, R. E. Woods, and S. L. Eddins.:'Digital Image Processing Using MATLAB', Prentice Hall, 2004.

[20] T. Master.:'Practical Neural Network Recipes'. New York: John Wiley, 1993. 